\title{
Strongly Enhanced Thermal Stability of Crystalline Organic Thin Films Induced by Aluminum Oxide Capping Layers
}

\author{
Stefan Sellner, ${ }^{1,2}$ Alexander Gerlach, ${ }^{3}$ Frank Schreiber, 3 , * Marion Kelsch, ${ }^{1}$ Nikolai Kasper,${ }^{1,4}$ \\ Helmut Dosch, ${ }^{1,2}$ Stephan Meyer, ${ }^{5}$ Jens Pflaum, ${ }^{5}$ Matthias Fischer, ${ }^{6}$ and Bruno Gompf ${ }^{6}$ \\ ${ }^{1}$ Max-Planck-Institut für Metallforschung, Heisenbergstr. 3, 70569 Stuttgart, Germany \\ ${ }^{2}$ Institut für Theoretische und Angewandte Physik, \\ Universität Stuttgart, Pfaffenwaldring 57, 70550 Stuttgart, Germany \\ ${ }^{3}$ Physical and Theoretical Chemistry Laboratory, Oxford University, South Parks Road, OX1 3QZ, United Kingdom \\ ${ }^{4}$ ANKA, FZ Karlsruhe, Hermann-von-Helmholtz Platz 1, 76344 Eggenstein-Leopoldshafen \\ ${ }^{5}$ III. Physikalisches Institut, Universität Stuttgart, Pfaffenwaldring 57, 70550 Stuttgart, Germany \\ ${ }^{6}$ I. Physikalisches Institut, Universität Stuttgart, Pfaffenwaldring 57, 70550 Stuttgart, Germany
}

(Dated: September 12, 2018)

\begin{abstract}
We show that the thermal stability of thin films of the organic semiconductor diindenoperylene (DIP) can be strongly enhanced by aluminum oxide capping layers. By thermal desorption spectroscopy and in-situ X-ray diffraction we demonstrate that organic films do not only stay on the substrate, but even remain crystalline up to $460^{\circ} \mathrm{C}$, i.e. $270^{\circ} \mathrm{C}$ above their desorption point for uncapped films $\left(190^{\circ} \mathrm{C}\right)$. We argue that this strong enhancement of the thermal stability compared to uncapped and also metal-capped organic layers is related to the very weak diffusion of aluminum oxide and the structurally well-defined as-grown interfaces. We discuss possible mechanisms for the eventual breakdown at high temperatures.
\end{abstract}

Keywords: organic thin films, heterostructures, thermal stability, X-ray reflectivity, thermal desorption spectroscopy

\section{INTRODUCTION}

Organic electronics is considered to be one of the key areas of future thin-film-device technology. Several device applications have already been shown to exhibit convincing performance, organic light-emitting diodes being one of the most successful examples [1, 2, 3]. However, besides the obvious performance requirements, the devices have to meet stability standards, which in some cases are actually the limiting factor of technological progress 4 . Indeed, stability at elevated temperatures, high electrical field gradients, and against exposure to corrosive gases like oxygen is crucial for all commercial applications.

It has turned out that thermal stability of thin organic films is not only related to fabrication procedures, but constitutes rather fundamental challenges 5 . It is thus a prerequisite to understand and to control 6 :

-interdiffusion at organic/metal interfaces during and after growth

-thermally induced dewetting effects at organic-inorganic interfaces

-structural phase transformations of the organic material at temperatures often not far from temperatures of operation

-the vapor pressure of low-weight organics at elevated temperatures.

Moreover, interfaces of organic films are often chemically and structurally heterogeneous, and their controlled

*corresponding author frank.schreiber@chem.ox.ac.uk preparation is non-trivial 7]. This applies to metallic contacts [8, 9, 10, 11], to insulating layers 12, 13], which are typically required in field-effect geometries, as well as to organic-organic interfaces as found in photovoltaic elements 1, 14].

In this paper we show that aluminum oxide layers can be prepared on organic semiconductor films of diindenoperylene (DIP) with well-defined interfaces which render thermally very stable heterostructures. This finding could open the way for organic thin film devices to operate at significantly higher temperatures than hitherto assumed.

\section{RESULTS}

\section{A. As-grown structure}

Thin films of DIP exhibit high structural quality 15, 16]. After depositing aluminum oxide on the organic film the TEM image (Fig. 1 $\mathrm{k}$ ) shows that the resulting heterostructure is very well-defined. Laterally homogeneous interfaces and only limited interdiffusion are observed. Within the organic film individual molecular layers of DIP can be resolved as indicated by the inset. The X-ray reflectivity data with pronounced Kiessig interferences - taken on a different sample - confirm this picture (Fig. 10). The Kiessig fringes correspond to a film thickness of $573 \AA$ for the aluminum oxide and $1020 \AA$ for the DIP film. Laue oscillations around the Bragg peak (see Fig. 3 below) of DIP confirm its high crystallinity, with the coherent thickness equaling the total thickness, 


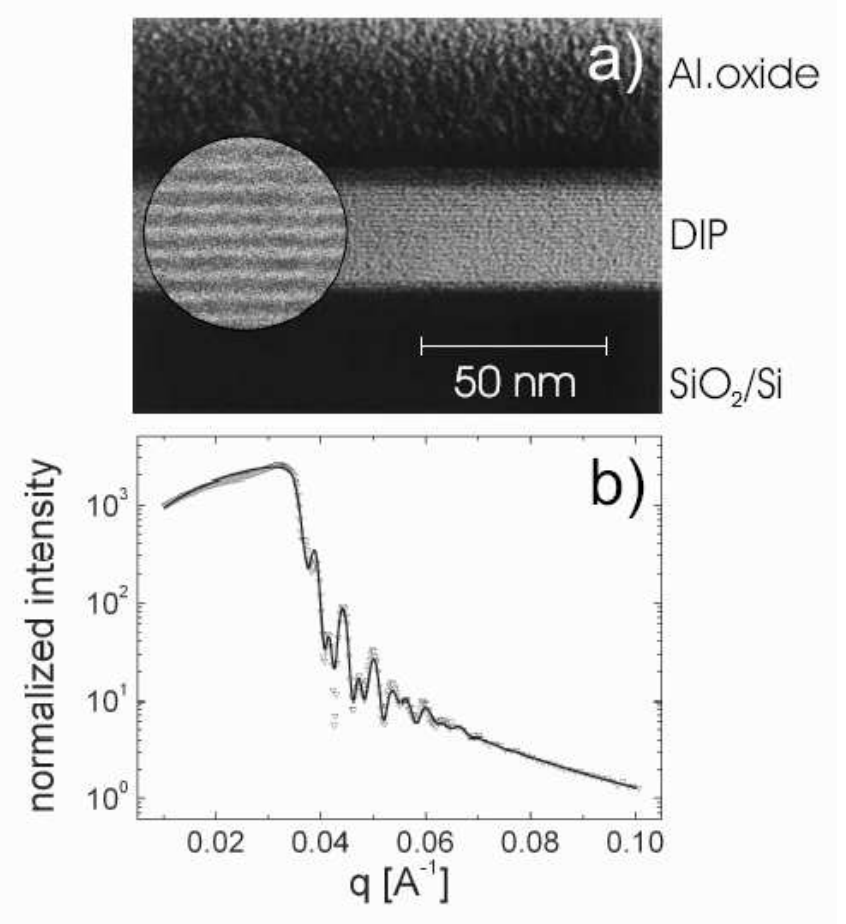

FIG. 1: a) TEM image showing a well-defined heterostructure of aluminum oxide on DIP (300 $)$ ) on silicon oxide together with the layer resolved structure of the organic film (inset). b) Room temperature X-ray reflectivity scan with least-square fit of an aluminum oxide capped DIP film showing pronounced Kiessig interference fringes.

implying that the DIP film is coherently ordered across its entire thickness. The well-defined character of the as-grown interfaces makes these heterostructures ideally suitable for studies of the thermal stability.

\section{B. Temperature dependence: Thermal desorption data}

Fig. 2 shows thermal desorption spectroscopy data which demonstrate the enhancement of the thermal stability of capped DIP films compared to uncapped layers. In these experiments the mass spectrometer is tuned to the mass of DIP molecules (400 amu), and the signal is recorded as a function of time, while the temperature is ramped at a constant rate of $0.5^{\circ} \mathrm{C} / \mathrm{sec}$. While the uncapped DIP shows a well-defined desorption peak around $190^{\circ} \mathrm{C}$ the capped film shows no desorption until $240^{\circ} \mathrm{C}$. The shape of the spectra also reveals differences in the desorption process. The spectrum of the uncapped film shows only one sharp peak that can be attributed to the DIP 'bulk' desorption. In contrast, the desorption spectrum of the capped film extends over a broader temperature range and has multiple features with the main feature centered at $300^{\circ} \mathrm{C}$.

We regard the sharp spikes as evidence for isolated des-

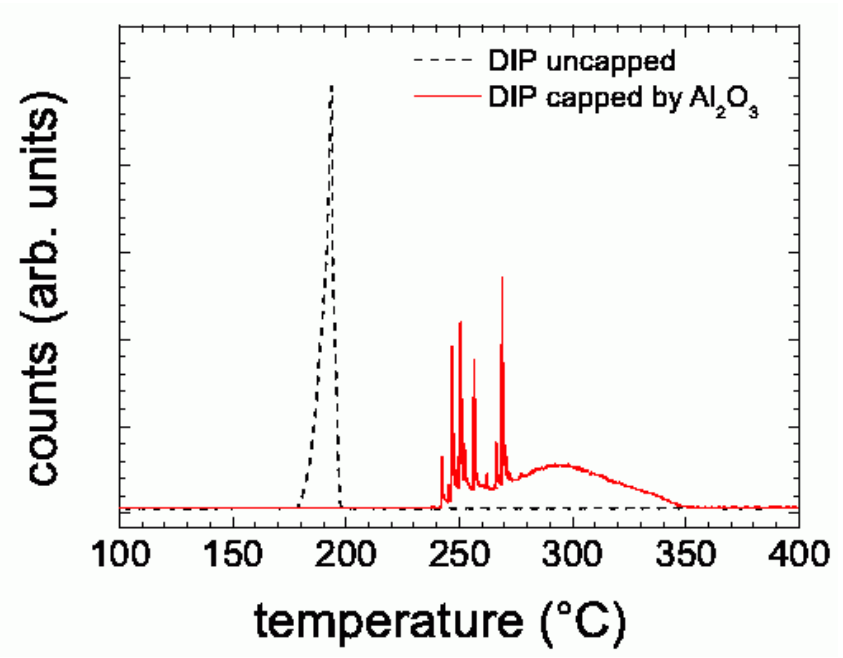

FIG. 2: Thermal Desorption Spectra of an uncapped vs. a capped DIP film. The uncapped DIP film (broken line) shows only one well-defined peak at $190^{\circ} \mathrm{C}$. The desorption features for the capped DIP film (solid line) are clearly shifted to higher temperatures.

orption channels such as small cracks, which do not give rise to desorption of the entire film. Only a small (local) fraction of DIP molecules may desorb at higher rate and on short time scales. The existence of these spikes was confirmed for several samples, but their exact position and height depends on the individual sample, consistent with the notion of the spikes being related to the properties of the individual capping layer. The broad desorption feature extending to high temperatures is related to the dominating part of the sample (the area under a given feature is proportional to the number of desorbing molecules). The wide temperature range may be taken as an indication of a laterally inhomogeneous capping layer and thus desorption processes from locally substantially different regions. Also, the state of the sample changes to some extent during the heating process, and one may speculate that the thermally stimulated desorption from a stable existing structure is also accompanied by a certain rate of thermally induced formation of cracks, and thus an increase of desorption from these defects which will depend on the exact heating rate. This is consistent with our finding that with decreasing heating rate the observed structural breakdown in TDS measurements shifts to higher temperatures.

\section{Temperature dependence: X-ray data}

In order to shed more light on the degradation process and the 'kinetics' of the breakdown at high temperatures we performed in-situ X-ray diffraction experiments (Fig. B). The films are heated up from $25^{\circ} \mathrm{C}$ to $500^{\circ} \mathrm{C}$ in a stepwise fashion and after thermal equilibration (on a time scale of a few minutes) X-ray reflectivity scans 

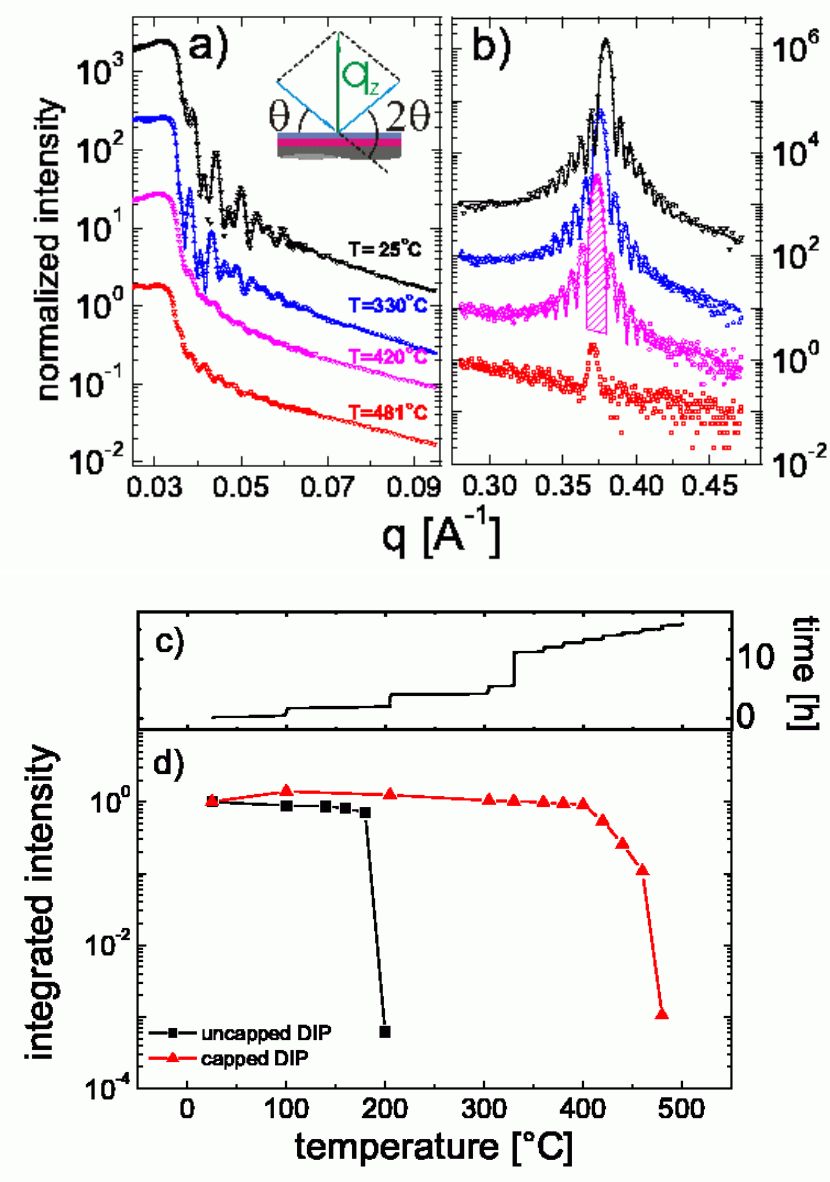

FIG. 3: (Upper panel) X-ray reflectivity data of the aluminum oxide/DIP heterostructure with least-square fits at different temperatures. The scattering geometry is indicated in the inset. By heating the sample the initially well-defined Kiessig fringes in panel (a) slowly degrade. The electron density profile can be reconstructed using the Parratt formalism 17] after correcting for diffuse scattering. Most prominently the roughness of the DIP/aluminum oxide and aluminum oxide/vacuum interfaces increase with higher temperatures. The first order Bragg peak with Laue oscillations in panel (b) remains visible up to $T=460^{\circ} \mathrm{C}$. For clarity the datasets are plotted with an offset. The comparison of the DIP film with and without aluminum oxide capping layer reveals the increased stability of the multilayer system as indicated by the integrated intensity of the Bragg peak as a function of temperature (d). For the temperature ramp in these experiments as displayed in panel (c) for the capped DIP film this results in a breakdown at $T=460^{\circ} \mathrm{C}$ compared to $T=190^{\circ} \mathrm{C}$ for uncapped DIP films.

(Fig. 3a and b) are taken at each intermediate temperature. Since the film is kept at elevated temperatures for several hours (see Fig. $3 \mathrm{k}$ ) the corresponding averaged 'heating rate' of $\sim 0.01^{\circ} \mathrm{C} / \mathrm{sec}$ is of course much lower than in the TDS experiment.

As can be seen from Fig. [3 $\mathrm{k}$, Kiessig interference fringes are clearly visible up to $\sim 380^{\circ} \mathrm{C}$, but are gradually damped out for still higher temperatures. Importantly, the Bragg reflection at $q \approx 0.38 \AA^{-1}$ (Fig. [3b) remains virtually unchanged up to $440^{\circ} \mathrm{C}$, showing that the DIP crystal structure stays intact. Until up to $460^{\circ} \mathrm{C}$, the Laue oscillations around the DIP Bragg peak show no significant changes implying that the organic film does not only remain crystalline, but also coherently ordered.

Fig. [3 compares the integrated intensity of the DIP Bragg peak with and without aluminum oxide capping layer for the given temperature-time protocol (Fig. 3k). As reported in previous studies [8, 9] and in agreement with TDS, uncapped DIP films desorb already at about $T=190^{\circ} \mathrm{C}$. In contrast, films with an aluminum oxide capping layer can be stabilized up to $T=460^{\circ} \mathrm{C}$, i.e. well beyond the desorption temperature of the uncapped DIP layer.

In a separate set of experiments, in order to evaluate long-term effects of heating, one reference sample was kept at $300^{\circ} \mathrm{C}$ and the DIP Bragg peak with Laue oscillations was recorded repeatedly for more than 300 hours. Its integrated intensity is found to decrease with time but exhibits still $\geq 50 \%$ of its initial value after $\sim 130$ hours - whereas the coherent thickness of the organic film remains unchanged over the entire period. This suggests that the observed degradation process of the capped DIP film is kinetically limited by desorption from defects within the capping layer. Since the coherent thickness stays constant the decrease of the integrated intensity at a certain temperature with time is related to desorption of the organic film from areas near thermally induced defects in the capping layer, as e.g. microcracks or holes. In a second step, even molecules from well-capped domains diffuse to these defects as a function of temperature and time (Fig. 四).

\section{DISCUSSION AND SUMMARY}

While an ideal capping layer is of course expected to suppress evaporation of the organic layer underneath, the remarkable finding is that, given the inevitable defects of real samples, the capping not only does enhance the stability, but does it so effectively. The increase of the thermal stability by up to $270^{\circ} \mathrm{C}$ in our experiments has to be seen in comparison with, e.g., metal capping layers which interdiffuse at low temperatures and tend to compromise the organic layer already upon deposition. We note that for accidentally non-stoichiometric aluminum oxide layers, specifically those with higher metal content, the DIP film structure broke down at temperatures in between $190^{\circ} \mathrm{C}$ and $450^{\circ} \mathrm{C}$. We take this as an indication of the metal atoms with their higher mobility being responsible for the weaker stabilization effect.

The oxide capping layer apparently is relatively near to the concept of a 'closed' layer and does not interdiffuse strongly upon deposition. Equally important, the amorphous aluminum oxide is less prone to diffusion than gold, so that even at elevated temperatures the capping layer presumably does not 'move' much, in contrast to gold, 

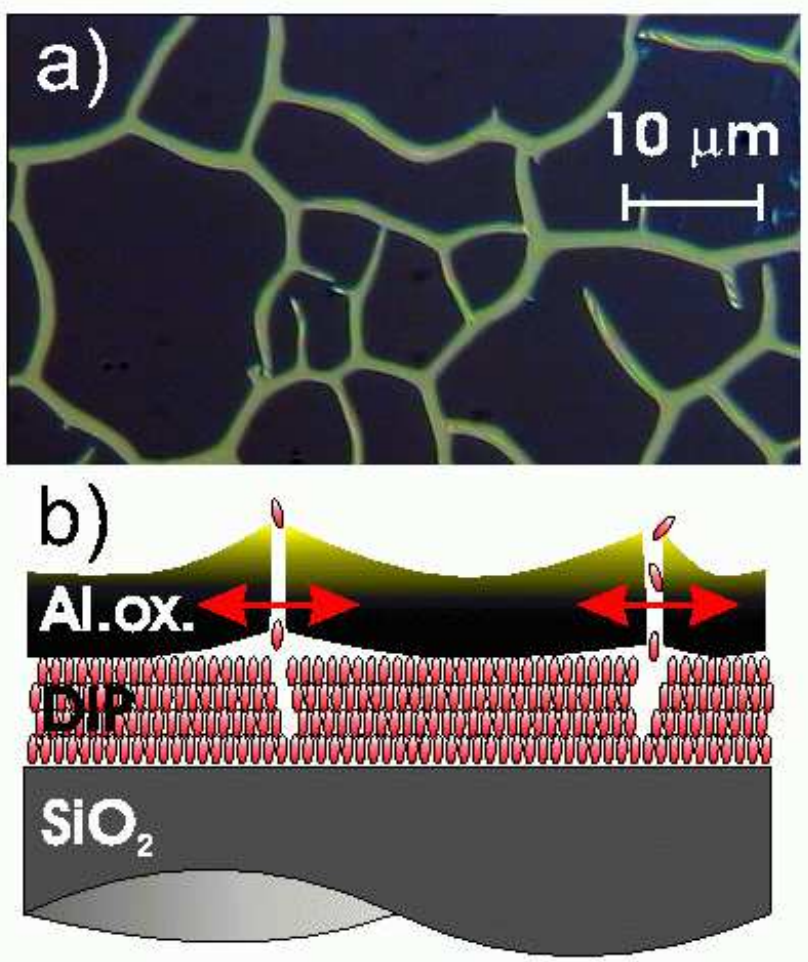

FIG. 4: a) Optical micrograph of the surface of the sample after heating. We interprete the bright lines as fractures in the capping layer induced by the thermal strain upon heating. b) Sketch of the degradation scenario resuming the experimental results.

which has a significant mobility at the temperatures relevant in this study. Nevertheless, also the oxide-capped DIP films ultimately break down. The detailed scenario of this process is still under investigation, but we speculate that it is related to minor cracks or narrow channels

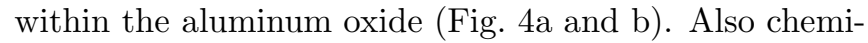
cal decomposition of DIP at high temperatures may play a role. Since the multilayer structure obviously depends strongly on the preparation conditions, the enhancement of the thermal stability will also vary accordingly.

The evidence of the enhanced thermal stability is of great practical importance, not only for the specific sys- tem of DIP studied here, but for organic electronics in general. It offers a route for the stabilization of compounds with vapor pressures so far considered too high for utilization in organic-based devices, thus extending the range of applications and working conditions, including harsher environments and elevated operating temperatures up to the thermal degradation of the molecules. The electrical characterization of capped semiconductors is presently underway.

\section{Experimental}

Thin films of DIP (thickness $300-1000 \AA$ ) on $\operatorname{Si}(100)$ wafers with native oxide were prepared by organic molecular beam deposition (rate $12 \AA / \mathrm{min}$, growth temperature $\left.145^{\circ} \mathrm{C}\right)[8,[15]$. The aluminum oxide layers were deposited on DIP by HF-magnetron sputtering in a separate chamber. To avoid oxidation of the underlying organic film we use pure argon as sputter gas (argon partial pressure $3 \times 10^{-3}$ mbar). After some sputter cycles this leads to an under-stoichiometric target with respect to oxygen content. To overcome this problem the target was refreshed after each deposition in an oxygen/argon atmosphere. The base pressure of the chamber was $3 \times 10^{-7} \mathrm{mbar}$, the deposition rate about $7 \AA / \mathrm{min}$ and the substrate temperature was kept at $-10^{\circ} \mathrm{C}$. Aluminum oxide films deposited under these conditions are totally transparent and amorphous.

The samples have then been studied by transmission electron microscopy (TEM), thermal desorption spectroscopy (TDS), and in-situ X-ray reflectivity at the Max-Planck beamline at the ANKA synchrotron source (FZ Karlsruhe, Germany). The data were taken from the centre of the sample so that edge effects can be excluded.

The temperature dependent X-ray reflectivity studies were done using a small vacuum chamber with a capton window and an integrated sample heater. All temperatures were measured with two calibrated C-type thermocouples close to the sample.

Acknowledgments We acknowledge support by the Deutsche Forschungsgemeinschaft (DFG) within the Focus Program on organic field effect transistors and by the Engineering and Physical Sciences Research Council (EPSRC). We are grateful to the FZ Karlsruhe and the ANKA management for their generous support.
[1] S. R. Forrest, Chem. Rev. 1997, 97, 1793

[2] P.K.H. Ho, J.S. Kim, J.H. Burroughes, H. Becker, S.F.Y Li, T.M. Brown, F. Cacialli, R.H. Friend, Nature 2000, 404, 481

[3] C. D. Dimitrakopoulos, P. R. L. Malenfant, Adv. Mater. 2002, 14, 99

[4] R. Farchioni and G. Grosso (Eds.), Organic Electronic Materials, Springer, Berlin 2001

[5] F. Schreiber, phys. stat. sol. (a) 2004, 201, 1037

[6] W. R. Salaneck, K. Seki, A. Kahn, J.-J. Pireaux, Conju- gated Polymer and Molecular Interfaces, Marcel Dekker, New York 2001

[7] P. Peumans, S. Uchida, and S. R. Forrest, Nature 2003, 425, 158

[8] A. C. Dürr, F. Schreiber, M. Kelsch, H. D. Carstanjen, H. Dosch, and O. H. Seeck, J. Appl. Phys. 2003, 93, 5201

[9] A. C. Dürr, F. Schreiber, M. Kelsch, H. D. Carstanjen, and H. Dosch, Adv. Mater. 2002, 14, 961

[10] N. Koch, A. C. Dürr, J. Ghijsen, R. L. Johnson, J.-J. Pireaux, J. Schwartz, F. Schreiber, H. Dosch, and A. 
Kahn, Thin Solid Films 2003, 441, 145

[11] F. Faupel, R. Willecke, and A. Thran, Mater. Sci. Eng. 1998, R22, 1

[12] J. H. Lee, S. H. Kim, G. H. Lim, and T. Zyung, Synthetic Metals 2003, 139, 445

[13] W. H. Ha, M. H. Choo, and S. Im, Journal of NonCrystalline Solids 2002, 303, 78

[14] P. Fenter, F. Schreiber, V. Bulovic, and S. R. Forrest, Chem. Phys. Lett. 1997, 27r, 521
[15] A. C. Dürr, F. Schreiber, M. Münch, N. Karl, B. Krause, V. Kruppa, and H. Dosch, Appl. Phys. Lett. 2002, 81, 2276

[16] A. C. Dürr, F. Schreiber, K. A. Ritley, V. Kruppa, J. Krug, H. Dosch, and B. Struth, Phys. Rev. Lett. 2003, 90, 016104

[17] M. Tolan, X-ray Scattering from Soft-Matter Thin Films, Springer, Berlin 1999 